\title{
Evidence of complement decay-accelerating factor transmembrane isoform gene of complement factor $b$ of complement factor $h$ genes in the sea star asterias rubens: three vertebrate regulators of complement pathways in an invertebrate
}

\begin{abstract}
The main components of Complement (alternative and direct pathway genes) correlated with mammal genes were discovered in the sea star genome. CR receptor gene was also found. We describe now the complement decay-accelerating factor transmembrane isoform gene: a regulator gene of Complement cascade. How genes are expressed? By which pathway? The presence of this last gene seems to indicate the direct pathway. But, on the other hand, Complement factor B gene was discriminated so that complement factor $\mathrm{H}$ gene in sea star genome, and these genes are implicated in alternative pathway. We may envisage that the two pathways co-exist in sea star immune response which leads to the formation of the sea star primitive antibody.
\end{abstract}

Volume 2 Issue 2 - 2017

\section{Michel Leclerc}

Doctor es Sciences University of Orléans, France

Correspondence: Michel Leclerc, Doctor es Sciences University of Orléans, 556 rue Isabelle Romée, Sandillon 45640, France,Email mleclerc45@gmail.com

Received: February 15, 2017| Published: February 23, 2017

Keywords: invertebrate, c1q subcomponent, complement c3, mouse complement factor $b$ gene

\section{Introduction}

Alternative and direct pathway genes correlated with mouse genes, were discovered in 2013:

C1q subcomponent subunit $\mathrm{A}, \mathrm{C} 1 \mathrm{q}$ subcomponent subunit $\mathrm{B}$, C1q subcomponent subunit $\mathrm{C}, \mathrm{C} 2$ component, Complement C4B, Complement C3, Complement component C9, Complement C5, Complement component C8 Alpha chain, were found Later CR receptor was discovered. ${ }^{2}$

How genes were expressed, by which pathway? The discovery of complement decay-accelerating factor transmembrane isoform gene, we study now, seems to indicate the direct pathway but Complement factor $\mathrm{H}$ gene and Complement factor B gene, we study also, indicate another pathway, as it was shown for mouse.

\section{Materials and methods}

Sea stars Asterias rubens were obtained from the Biology Institute (Gothenburg University) Immunizations to HRP (Horse-radish peroxydase) and genomic studies were already described. ${ }^{1}$

\section{Results and discussion}

Mouse complement decay-accelerating factor transmembrane isoform gene was found in non-immunized animals to HRP:

Control: Contig11310 sp|Q61476|DAF2_MOUSE Complement decay-accelerating factor transmembrane isoform $\mathrm{OS}=$ Mus musculus $\mathrm{GN}=\mathrm{Cd} 55 \mathrm{~b} \quad \mathrm{PE}=2 \mathrm{SV}=2$

Mouse complement factor B gene was discriminated in control and immunized sea stars to HRP:
HRP: $\quad$ Contig16117|m.12266 sp|P04186|CFAB MOUSE Complement factor $\mathrm{B}$ OS=Mus musculus $\mathrm{GN}=\mathrm{Cfb} \mathrm{PE}=1 \mathrm{SV}=2$

Mouse complement factor $\mathrm{H}$ was also discriminated in nonimmunized sea stars to HRP:

Control: Contig9673 sp|P06909|CFAH_MOUSE Complement factor $\mathrm{H}$ OS=Mus musculus $\mathrm{GN}=\mathrm{Cfh} \mathrm{PE}=1 \mathrm{SV}=2$

Activation of Complement via the alternative pathway ${ }^{3}$ represents one means of natural infection, in Mouse; it implies six serum proteins. The reaction initiates $\mathrm{C} 3 \mathrm{~b}$, factors $\mathrm{H}$ and $\mathrm{B}$ which are present in sea star genome.

On the other hand the classical pathway is activated by an antigenantibody reaction which has been also described in sea star immune model; it implies C1q mainly then $\mathrm{C} 4, \mathrm{C} 2$, found in sea star genome. These genes are mainly vertebrate genes. So the alternative pathway and classical one are present and could co-exist in the sea star immune system, especially in the antigen-antibody reaction.

\section{Acknowledgements}

None.

\section{Conflict of interest}

The author declares no conflict of interest.

\section{References}
1. Leclerc M. Amer J Immunol. 2013;9(1):26-29.
2. Leclerc M. Int J Cell Sci. 2016;1(1):000105.
3. Panqburn MK. Fed Proc. 1983;42(1):139-143. 